\title{
Propyl gallate inhibits TPA-induced inflammation via the nuclear factor-kB pathway in human THP-1 monocytes
}

\author{
HUNG-CHIH HSU ${ }^{1-3}$, WAN-CHEN LIN ${ }^{1,4}$, PEY-JIUM CHANG ${ }^{2}$, CHANG-ZERN HONG ${ }^{5}$ and CHING-HSEIN CHEN ${ }^{4}$ \\ ${ }^{1}$ Department of Physical Medicine and Rehabilitation, Chang Gung Memorial Hospital, Chiayi; ${ }^{2}$ Graduate Institute \\ of Clinical Medical Sciences, College of Medicine, Chang Gung University, Taoyuan; ${ }^{3}$ Department of Nursing, \\ Chang Gung University of Science and Technology, Chiayi; ${ }^{4}$ Department of Microbiology, \\ Immunology and Biopharmaceuticals, College of Life Sciences, National Chiayi University, Chiayi; \\ ${ }^{5}$ Department of Physical Therapy, Hung Kuang University, Taichung, Taiwan, R.O.C.
}

Received September 26, 2012; Accepted January 10, 2013

DOI: $10.3892 /$ etm.2013.896

\begin{abstract}
Propyl gallate (PG) is an antioxidant that has been used as an additive in several foods to protect against oxidation. The present study examined the anti-inflammatory effect of PG on 12-O-tetradecanoylphorbol-13-acetate (TPA)-induced inflammation in human THP-1 monocytes. Pretreatment with PG markedly inhibited the TPA-induced expression levels of cyclooxygenase-2 and prostaglandin E2. The application of PG significantly inhibited the nuclear translocation of p65, a subunit of nuclear factor- $\kappa \mathrm{B}(\mathrm{NF}-\kappa \mathrm{B})$ and phosphorylation of p65 (Ser536) in TPA-treated THP-1 cells. PG also inhibited the phosphorylation of $\mathrm{I} \kappa \mathrm{B}$ and $\mathrm{I} \kappa \mathrm{B}$ kinase. These results indicate that $\mathrm{PG}$ inhibits the inflammatory response by blocking the NF- $\kappa \mathrm{B}$ signaling pathway in TPA-induced THP-1 monocytes. Therefore, PG may be useful as a therapeutic agent in inflammatory diseases.
\end{abstract}

\section{Introduction}

Propyl gallate (PG), an ester that is also known as propyl 3,4,5-trihydroxybenzoate and belongs to the polyphenolic compound family, is synthesized by the condensation of propanol and gallic acid. PG is used in foods, cosmetics, hair products, adhesives and lubricants due to its antioxidative properties, which protect foods containing oils and fats from oxidation by hydrogen peroxide and free oxygen radicals.

The formation, metabolism, catabolism, and physiological and pathophysiological roles of prostanoids are associated with the development of inflammation and carcinogenesis (1).

Correspondence to: Professor Ching-Hsein Chen, Department of Microbiology, Immunology and Biopharmaceuticals, College of Life Sciences, National Chiayi University, 300 Syuefu Road, Chiayi 60004, Taiwan, R.O.C.

E-mail: chench@mail.ncyu.edu.tw

Key words: propyl gallate, anti-inflammation, cyclooxygenase-2, nuclear factor- $\kappa \mathrm{B}$
Cyclooxygenase-2 (COX-2) is an enzyme that is critical for prostanoid synthesis (2). This process is initiated by the enzymatic release of arachidonic acid (AA) from cellular stores by phospholipase A2 (3). AA is then metabolized to prostaglandins via the COX-2 pathway through a series of enzymatic steps (4). COX-2 is inducible and may be associated with one or more pathophysiological states or reactions (5). Since a number of the metabolic steps in this pathway generate reactive oxygen species (6), it is important to develop COX-2-specific agents that are effective anti-inflammatory drugs or have cancer preventive activity.

The discovery of the molecular associations between inflammation and cancer was a major breakthrough in chemoprevention research. Components of the cell signaling network have been implicated in the promotional stage of carcinogenesis, particularly those that converge on the redox-sensitive transcription factor nuclear factor $-\kappa \mathrm{B}(\mathrm{NF}-\kappa \mathrm{B})$, which is involved in mediating the inflammatory response $(7,8)$. One of the major target molecules subjected to NF- $\mathrm{B}$-driven transactivation is COX-2, which is involved in the biosynthesis of prostaglandins and inflammation (9). Inappropriate upregulation of COX-2 has frequently been observed in various premalignant and malignant tissues (10). The role of abnormally high levels of COX-2 in tumorigenesis has further been corroborated by the increased susceptibility of COX-2-overexpressing mice (11) and the relatively increased resistance of COX-2-knockout animals to spontaneous or experimentally induced carcinogenesis (12). Therefore, targeted inhibition of COX-2 is regarded as a promising and practical approach to prevent cancer (13).

$\mathrm{NF}-\kappa \mathrm{B}$ generally exists as a heterodimer of the p50 and RelA (p65) polypeptides, bound in an inactive state in the cytoplasm by the inhibitor protein $\mathrm{I} \kappa \mathrm{B}(14)$. Following cellular stimulation by a variety of agents, I $\mathrm{I} B$ is phosphorylated and degraded by the proteasome, allowing NF- $\kappa \mathrm{B}$ to translocate to the nucleus and regulate the expression of the target genes, including a number that control cellular growth properties and apoptotic cell death $(15,16)$. The transactivation of $\mathrm{NF}-\kappa \mathrm{B}$-regulated genes requires not only the binding of $\mathrm{NF}-\kappa \mathrm{B}$ to the promoter regions, but also the phosphorylation 


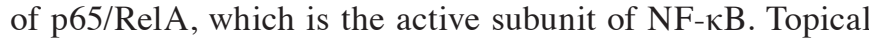
applications of 12-O-tetradecanoylphorbol-13-acetate (TPA) have been shown to cause an increase in p65/RelA phosphorylation at serine 536 (17).

PG is an antioxidant which affects and/or inhibits the inflammatory pathway. Although it appears that the inhibition of TPA-induced COX-2 by PG occurs through the NF- $\mathrm{BB}$ pathway, the details of the mechanism remain to be elucidated. In the present study, TPA-induced COX-2 was used in human THP-1 cells as an inflammation model to evaluate the effect of PG on COX-2 expression and prostaglandin E2 (PGE2) production. The effect of $\mathrm{PG}$ on the $\mathrm{NF}-\kappa \mathrm{B}$ pathway was also determined.

\section{Materials and methods}

Cell line and reagents. The human THP-1 cell line was obtained from the Bioresource Collection and Research Center (Hsinchu, Taiwan, R.O.C.). Roswell Park Memorial Institute-1640 (RPMI-1640) and fetal bovine serum (FBS) were obtained from Hyclone (South Logan, UT, USA). Anti-COX-2, anti-NF- $\kappa$ B, anti-I $\kappa$ B, anti-IKK $\alpha$ and anti- $\beta$-actin primary antibodies and a secondary antibody labeled with horseradish-peroxidase were purchased from Santa Cruz Lab Vision (Santa Cruz, CA, USA). Anti-phospho-IKK $\alpha$ (Ser180)/IKK $\beta$ (Ser181), anti-p65/RelA and anti-phospho-p65 (Ser536) primary antibodies were purchased from Cell Signaling Technology, Inc. (Danvers, MA, USA). PG, TPA and other chemicals were purchased from Sigma Chemical Co. (St. Louis, MO, USA) and the PGE2 enzyme immunoassay kit was purchased from Stressgen (Ann Arbor, MI, USA).

Cell culture and treatment. The basal medium for the THP-1 cell line culture was composed of RPMI-1640 supplemented with $10 \% \mathrm{FBS}, 100 \mathrm{U} / \mathrm{ml}$ penicillin $\mathrm{G}$ and $100 \mu \mathrm{g} / \mathrm{ml}$ streptomycin. The PG stock solution $(100 \mathrm{mM})$ was dissolved in dimethylsulfoxide (DMSO) and various concentrations were prepared in the basal medium with a final DMSO concentration of $0.1 \%$, which was considered to cause little or no damage in the THP-1 cells.

PGE2 assay. A PGE2 EIA kit from Stressgen was used to measure the PGE2 secreted in conditioned cell culture media of serum-starved cells for $24 \mathrm{~h}$ with and without TPA or PG, according to the manufacturer's instructions.

Western blot analysis. Following treatment, cells were washed with phosphate-buffered saline (PBS), resuspended in a protein extraction buffer for $10 \mathrm{~min}$, then centrifuged at $12,000 \mathrm{rpm}$ for $10 \mathrm{~min}$ at $4^{\circ} \mathrm{C}$ to obtain the total extracted proteins. The protein concentrations were evaluated with a Bio-Rad protein assay reagent (Bio-Rad, Richmond, CA, USA). The expression levels of various intracellular proteins were then evaluated by western blot analyses. Briefly, the total extracted protein content was boiled in a loading buffer and an aliquot corresponding to 50 or $100 \mu \mathrm{g}$ protein was separated by $12 \%$ SDS-polyacrylamide gel. Following electrophoresis, proteins were electrotransferred onto a polyvinylidene fluoride transfer membrane. After blotting, the membranes were incubated with various primary antibodies overnight at $4^{\circ} \mathrm{C}$
A

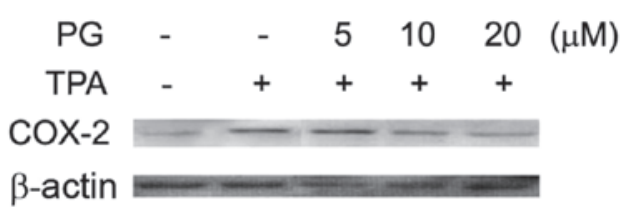

B

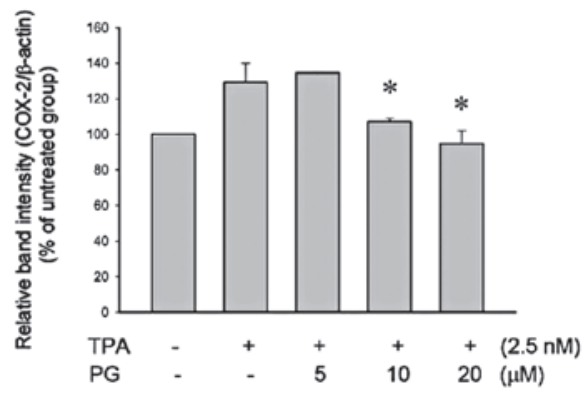

Figure 1. Inhibitory effects of PG on TPA-induced COX-2 protein levels in THP-1 cells. (A) The cells were treated with PG $(5-20 \mu \mathrm{M})$ for $2 \mathrm{~h}$ prior to treatment with $2.5 \mathrm{nM}$ TPA for $24 \mathrm{~h}$. (A) COX-2 expression was evaluated by western blotting; (B) COX-2 expression levels were normalized to $\beta$-actin using a densitometer. ${ }^{*} \mathrm{P}<0.05$ represents a significant difference from the cells treated with TPA only; $(n=3)$. PG, propyl gallate; TPA, 12-O-tetradecanoylphorbol-13-acetate; COX-2, cyclooxygenase-2.

and then washed with PBST solution $(0.05 \%$ Tween-20 in PBS). Following washing, the secondary antibody, labeled with horseradish-peroxidase, was incubated for $1 \mathrm{~h}$ at room temperature and then washed with the PBST solution. The antigen-antibody complexes were detected by enhanced chemiluminescence using a chemiluminescence analyzer (Amersham Pharmacia Biotech, Piscataway, NJ, USA).

Statistical analysis. The data are presented as the mean \pm standard deviation from at least 3 independent experiments and were analyzed using Student's t-tests. $\mathrm{P}<0.05$ was considered to indicate a statistically significant difference.

\section{Results}

PG inhibited TPA-induced COX-2 in THP-1 cells. TPA is an inflammatory agent that targets intracellular protein kinase $C$ (PKC) and induces inflammation via the activation of the $\mathrm{NF}-\kappa \mathrm{B}$ pathway. In order to evaluate the anti-inflammatory activity of PG, TPA was used to induce inflammation in THP-1 cells. Treatment with $2.5 \mathrm{nM}$ TPA for $24 \mathrm{~h}$ increased COX-2 expression levels in the THP-1 cells. Cells that were pretreated with PG $(10$ or $20 \mu \mathrm{M})$ for $2 \mathrm{~h}$ before the TPA treatment showed significantly inhibited COX-2 expression (Fig. 1).

PG inhibited TPA-induced PGE2 in THP-1 cells. PGE2 is an extensively studied prostaglandin owing to its predominance in inflammation. PGE2 has been of great interest as a therapeutic target, for example, through the modulation of its synthesis by COX inhibitors. To evaluate whether PG suppresses PGE2 production in human monocytes treated with inflammatory agents, human THP-1 monocyte cell lines were pretreated with PG (5-20 $\mu \mathrm{M})$ for $2 \mathrm{~h}$ and then exposed to $2.5 \mathrm{nM}$ TPA 


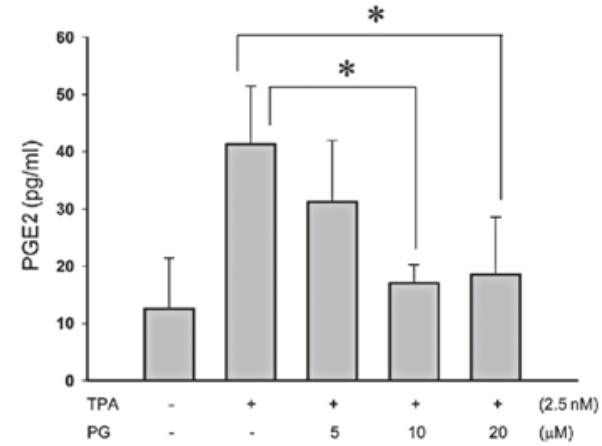

Figure 2. Effect of PG on TPA-induced prostaglandin PGE2 release from THP-1 cells. The cells were pretreated with PG for $2 \mathrm{~h}$, then stimulated by $2.5 \mathrm{nM}$ TPA for $48 \mathrm{~h}$. ${ }^{*} \mathrm{P}<0.05$ represents a significant difference from the cells treated with TPA only. PG, propyl gallate; PGE2, prostaglandin E2; TPA, 12-O-tetradecanoylphorbol-13-acetate.

A

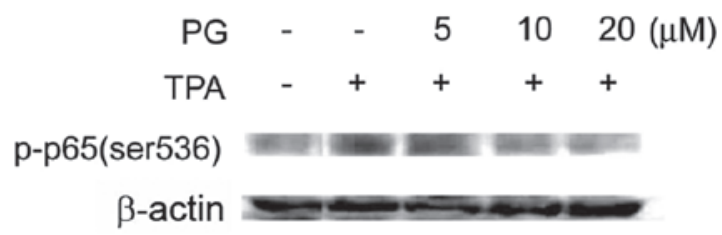

B

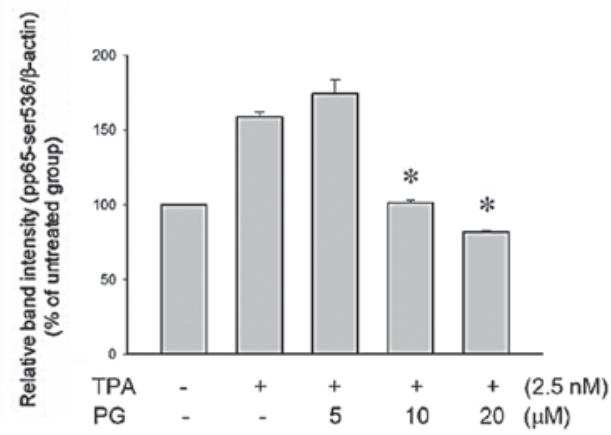

Figure 3. Inhibitory effects of PG on TPA-induced phosphorylation of p65 (Ser536) in THP-1 cells. The cells were pretreated with PG $(5,10$ or $20 \mu \mathrm{M})$ for $2 \mathrm{~h}$, then incubated with $2.5 \mathrm{nM}$ TPA for $2.5 \mathrm{~h}$. (A) p-p65 expression was evaluated by western blotting; (B) p-p65 (Ser536) expression levels were normalized to $\beta$-actin using a densitometer. ${ }^{*} \mathrm{P}<0.05$ represents a significant difference from the cells treated with TPA only; $(n=3)$. PG, propyl gallate; TPA, 12-O-tetradecanoylphorbol-13-acetate.

for $48 \mathrm{~h}$. Using a PGE2 ELISA kit, it was demonstrated that pretreatment with 10 or $20 \mu \mathrm{M}$ PG significantly inhibited PGE2 production (Fig. 2). These results suggest that PG has anti-inflammatory activity.

$P G$ inhibited TPA-induced activation of $N F-\kappa B$ and nuclear translocation in THP-1 cells. NF- $\mathrm{KB}$, a component of the cell signaling network, is a redox-sensitive transcription factor involved in the mediation of the inflammatory response. One of the major target molecules subjected to NF- $\mathrm{kB}$-driven transactivation is COX-2, which is involved in the biosynthesis of prostaglandins and inflammation (9). Therefore, whether the observed anti-inflammatory activity of PG occurred via NF- $\kappa \mathrm{B}$ signaling was investigated. The transactivation
A

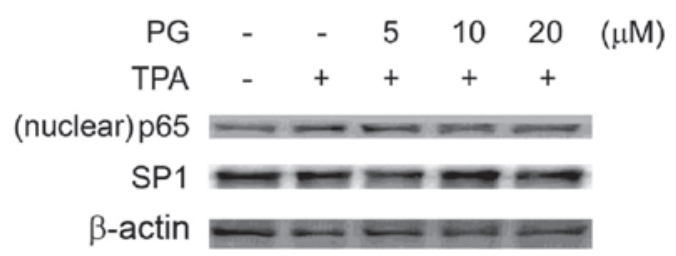

B

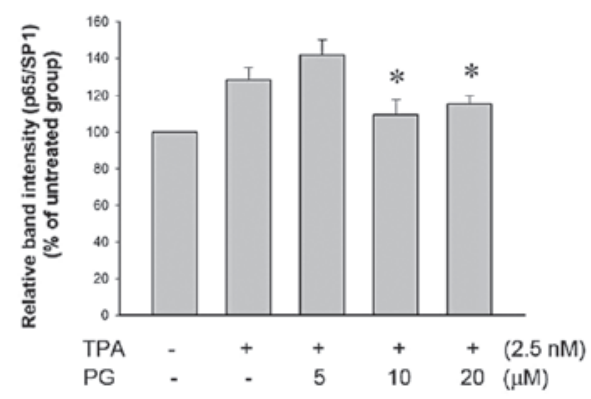

Figure 4. Inhibitory effects of PG on TPA-induced p65 nuclear translocation in THP-1 cells. The cells were pretreated with PG $(5,10$ or $20 \mu \mathrm{M})$ for $2 \mathrm{~h}$, then incubated with $2.5 \mathrm{nM}$ TPA for $4 \mathrm{~h}$. (A) p65 expression in the nucleus was evaluated by western blotting; (B) p65 expression levels were normalized to $\beta$-actin using a densitometer. ${ }^{*} \mathrm{P}<0.05$ represents a significant difference from the cells treated with TPA only; $(n=3)$. PG, propyl gallate; TPA, 12-O-tetradecanoylphorbol-13-acetate.

A

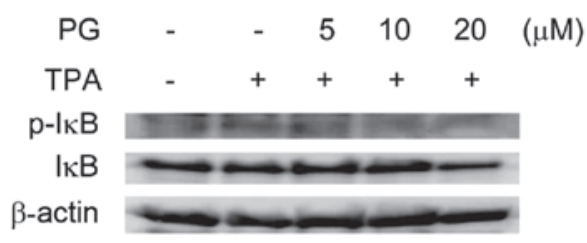

B

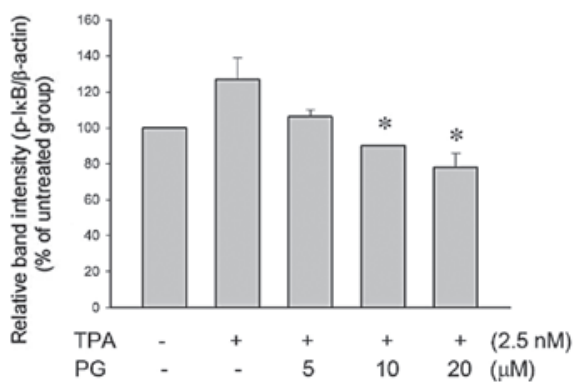

Figure 5. Inhibitory effects of PG on TPA-induced phosphorylation of I $\mathrm{B}$ in THP-1 cells. The cells were pretreated with PG $(5,10$ or $20 \mu \mathrm{M})$ for $2 \mathrm{~h}$, then incubated with $2.5 \mathrm{nM}$ TPA for $2.5 \mathrm{~h}$. (A) $\mathrm{p}$-IкB expression was evaluated by western blotting; (B) p-IкB expression was normalized to $\beta$-actin using a densitometer. " $\mathrm{P}<0.05$ represents a significant difference from the cells treated with TPA alone; $(n=3)$. PG, propyl gallate; TPA, 12-O-tetradecanoylphorbol-13-acetate.

of NF- $\kappa \mathrm{B}$-regulated genes requires the phosphorylation of p65/RelA (p-p65), the active subunit of NF- $\mathrm{kB}$. The cells were pretreated with PG $(5,10$ or $20 \mu \mathrm{M})$ for $2 \mathrm{~h}$ followed by incubation with TPA $(2.5 \mathrm{nM})$ for $2.5 \mathrm{~h}$. The phosphorylation of $\mathrm{p} 65 /$ RelA (Ser536) was significantly inhibited by treatment with 
A

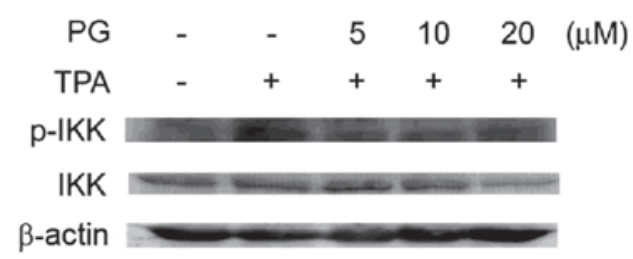

B

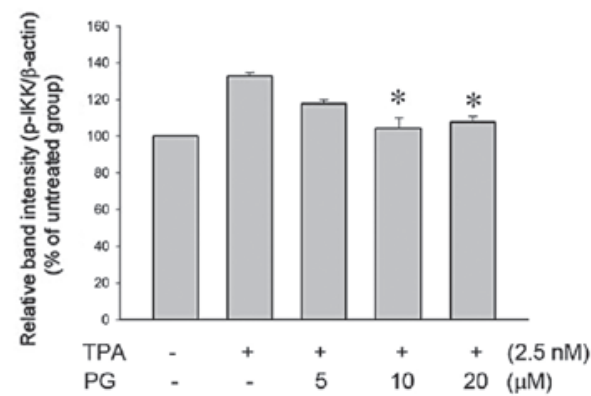

Figure 6. Inhibitory effects of PG on TPA-induced activation of p-IKK protein expression in THP- 1 cells. The cells were pretreated with PG $(5,10$ or $20 \mu \mathrm{M}$ ) for $2 \mathrm{~h}$, then incubated with $2.5 \mathrm{nM}$ TPA for $30 \mathrm{~min}$. (A) p-IKK expression was evaluated by western blotting; (B) p-IKK expression levels were normalized to $\beta$-actin using a densitometer. ${ }^{*} \mathrm{P}<0.05$ represents a significant difference from the group treated with TPA only; $(n=3)$. PG, propyl gallate; TPA, 12-O-tetradecanoylphorbol-13-acetate; IKK, IкB kinase.

10 or $20 \mu \mathrm{M}$ PG (Fig. 3). In addition, treatment with these two concentrations of PG (10 and $20 \mu \mathrm{M})$ significantly inhibited the nuclear translocation of p65 (Fig. 4), indicating that the anti-inflammatory activity of PG occurred via the inhibition of the NF- $\mathrm{kB}$ signaling pathway.

$P G$ inhibited TPA-induced phosphorylation of I $\kappa B$ in THP-1 cells. NF- $\mathrm{\kappa B}$ is bound in an inactive state in the cytoplasm by the inhibitor protein I $\mathrm{I} B$ (14). Following cellular stimulation by a variety of agents, I $\kappa \mathrm{B}$ is phosphorylated and then degraded by the proteasome, allowing NF- $\kappa \mathrm{B}$ to translocate to the nucleus and regulate the expression of the target genes. The phosphorylation of IкB was increased by the TPA treatment (Fig. 5), indicating that TPA activates NF- $\mathrm{kB}$ signaling via the phosphorylation of

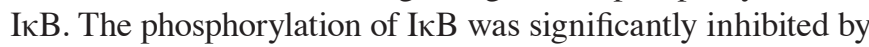
treatment with 10 or $20 \mu \mathrm{M} \mathrm{PG}$ (Fig. 5).

$P G$ inhibited TPA-induced phosphorylation of I $1 \kappa B$ kinase (IKK) in THP-1 cells. IKK is able to phosphorylate IкB and cause its degradation by ubiquitination. It was further evaluated whether PG was able to inhibit the IKK activity in TPA-treated THP-1 cells. As shown in Fig. 6, the PG treatment (10 or $20 \mu \mathrm{M}$ ) significantly inhibited the phosphorylation of IKK. The expression of IKK was also inhibited by treatment with $20 \mu \mathrm{M}$ PG. These results indicated that PG inhibits the

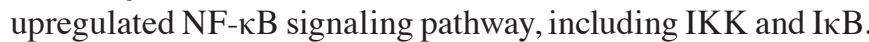

\section{Discussion}

In anti-inflammatory studies, three general categories of inflammatory inducers are considered including, i) gram-negative bacterial cell wall lipopolysaccharide (LPS); ii) proinflamma- tory cytokines such as tumor necrosis factors (TNF- $\alpha$ ); and iii) the tumor promoter agent TPA. With LPS, the cells are able to simulate pathogenic infections caused by inflammation. TNF- $\alpha$ directly binds to specific receptors (TNFR1 and TNFR2), which then activate the NF- $\mathrm{KB}$ signaling pathway to induce inflammation. TPA penetrates intracellularly and activates PKC and thus, the NF- $\mathrm{KB}$ signaling pathway is activated to induce inflammation. Compared with LPS, TPA is chemically more stable, less variable and requires a lower concentration to induce inflammation. Furthermore, unlike TNF- $\alpha$, TPA does not induce caspase activation. Therefore, in the present study, TPA was selected as the inflammation inducer.

Numerous antioxidants extracted from food show excellent anti-inflammatory effects. For example, piperine, a major component of black (Piper nigrum Linn) and long (Piper longum Linn) pepper, exhibits antioxidant activity. The dose-dependent decrease of phorbol 12-myristate 13-acetate (PMA)-induced COX-2 expression and PGE2 production in murine RAW 264.7 macrophages by piperine was identified to be partially due to the inhibition of PMA-induced NF- $\mathrm{KB}$ nuclear translocation (18). The dietary flavonoid quercetin is an antioxidant that possesses anti-inflammatory properties. Quercetin protects cells against TNF- $\alpha$-induced activation of the NF- $\mathrm{BB}$ signaling pathway (19). This inhibitory effect of quercetin was mediated, at least in part, by extracellular regulated kinase, c-jun amino-terminal kinase and reactive oxygen species and accompanied by reduced COX-2 levels (19).

It is notable that tannin and phenolic compounds extracted from tomatoes express excellent inhibition effects in TPA-induced COX-2 expression in KB cells (20). These results are in agreement with those of the present study showing that the anti-inflammatory effect of PG occurs via the NF- $\kappa B$ signaling pathway and COX-2 inhibition in TPA-treated THP-1 cells. PG belongs to the polyphenolic compound family and is synthesized by the condensation of propanol and gallic acid. In general, PG has been proposed to act as an antioxidant that protects foods against oxidation by hydrogen peroxide and oxygen free radicals. It is used in foods, cosmetics, hair products, adhesives and lubricants. Our present study shows that apart from its antioxidant activity, PG also demonstrates anti-inflammatory activity.

A number of studies have reported that gallic acid (3,4,5-trihydroxybenzoic acid), a natural polyphenol obtained from gallnuts and green tea, has antioxidant, anti-inflammatory and radical-scavenging activities. In human mast cells, gallic acid decreased the PMA plus calcium ionophore A23187-stimulated gene expression and production of proinflammatory cytokines such as TNF- $\alpha$ and inteleukin (IL)-6. The inhibitory effect of gallic acid on the proinflammatory cytokines was demonstrated to be dependent on NF- $\mathrm{KB}$ and p38 mitogen-activated protein kinase (21). Our previous study demonstrated that the antioxidant activity of PG is higher than that of gallic acid in THP-1 cells treated with various reactive oxygen species (22). Another study has demonstrated that certain derivatives of gallic acid exhibit good anti-inflammatory activity, as determined by the carrageenan-induced paw edema test (23). These studies may provide an explanation for the present results, wherein PG inhibits TPA-induced inflammatory reactions in THP-1 cells by blocking COX-2, PGE2 and IKK activity and NF- $\mathrm{KB}$ 
signaling and these effects may partially be associated with its excellent antioxidant activity.

\section{Acknowledgements}

This study was supported in part by grants from the National Science Council (NSC 96-2320-B-415-002-MY3, C.H.C.) and Chang Gung Memorial Hospital, R.O.C. (CMRPG6A0291, H.C.H.).

\section{References}

1. Mutoh M, Takahashi M and Wakabayashi K: Roles of prostanoids in colon carcinogenesis and their potential targeting for cancer chemoprevention. Curr Pharm Des 12: 2375-2382, 2006.

2. Piston D, Wang S, Feng Y, et al: The role of cyclooxygenase-2/prostanoid pathway in visceral pain induced liver stress response in rats. Chin Med J (Engl) 120: 1813-1819, 2007.

3. Weerasinghe GR, Coon SL, Bhattacharjee AK, et al: Regional protein levels of cytosolic phospholipase A2 and cyclooxygenase-2 in Rhesus monkey brain as a function of age. Brain Res Bull 69: 614-621, 2006.

4. Nagasaki S, Suzuki T, Miki Y, et al: 17Beta-hydroxysteroid dehydrogenase type 12 in human breast carcinoma: a prognostic factor via potential regulation of fatty acid synthesis. Cancer Res 69: 1392-1399, 2009.

5. Branski RC, Zhou H, Sandulache VC, et al: Cyclooxygenase-2 signaling in vocal fold fibroblasts. Laryngoscope 120: 1826-1831, 2010.

6. Oláh O, Németh I, Tóth-Szüki V, et al: Regional differences in the neuronal expression of cyclooxygenase-2 (COX-2) in the newborn pig brain. Acta Histochem Cytochem 45: 187-192, 2012.

7. Slattery ML, Lundgreen A, Bondurant KL and Wolff RK: Interferon-signaling pathway: associations with colon and rectal cancer risk and subsequent survival. Carcinogenesis 32: 1660-1667, 2011

8. Setia S and Sanyal SN: Nuclear factor kappa B: a pro-inflammatory, transcription factor-mediated signalling pathway in lung carcinogenesis and its inhibition by nonsteroidal anti-inflammatory drugs. J Environ Pathol Toxicol Oncol 31: 27-37, 2012.

9. Liu D, Kim DH, Park JM, et al: Piceatannol inhibits phorbol ester-induced NF-kappa B activation and COX-2 expression in cultured human mammary epithelial cells. Nutr Cancer 61 : 855-863, 2009.

10. Fernández-Martínez AB, Carmena MJ, Arenas MI, et al: Overexpression of vasoactive intestinal peptide receptors and cyclooxygenase- 2 in human prostate cancer. Analysis of potential prognostic relevance. Histol Histopathol 27: 1093-1101, 2012.
11. Muller-Decker K, Neufang G, Berger I, et al: Transgenic cyclooxygenase-2 overexpression sensitizes mouse skin for carcinogenesis. Proc Natl Acad Sci USA 99: 12483-12488, 2002.

12. Tiano HF, Loftin CD, Akunda J, et al: Deficiency of either cyclooxygenase (COX)-1 or COX-2 alters epidermal differentiation and reduces mouse skin tumorigenesis. Cancer Res 62: 3395-3401, 2002.

13. Cerella C, Sobolewski C, Chateauvieux S, et al: COX-2 inhibitors block chemotherapeutic agent-induced apoptosis prior to commitment in hematopoietic cancer cells. Biochem Pharmacol 82: 1277-1290, 2011.

14. Gutierrez H, O'Keeffe GW, Gavaldà N, et al: Nuclear factor kappa B signaling either stimulates or inhibits neurite growth depending on the phosphorylation status of p65/RelA. J Neurosci 28: 8246-8256, 2008

15. Li X, Wu G, Wu M, et al: In vitro study of inhibitory millimeter wave treatment effects on the TNF- $\alpha$-induced NF- $\mathrm{BB}$ signal transduction pathway. Int J Mol Med 27: 71-78, 2011.

16. Kole L, Giri B, Manna SK, et al: Biochanin-A, an isoflavon, showed anti-proliferative and anti-inflammatory activities through the inhibition of iNOS expression, p38-MAPK and ATF-2 phosphorylation and blocking NFKB nuclear translocation. Eur J Pharmacol 653: 8-15, 2011.

17. Kim SO, Kundu JK, Shin YK, et al: [6]-Gingerol inhibits COX-2 expression by blocking the activation of p38 MAP kinase and NF-kappaB in phorbol ester-stimulated mouse skin. Oncogene 24: 2558-2567, 2005.

18. Kim HG, Han EH, Jang WS, et al: Piperine inhibits PMA-induced cyclooxygenase- 2 expression through downregulating NF- $\kappa \mathrm{B}$, $\mathrm{C} / \mathrm{EBP}$ and AP-1 signaling pathways in murine macrophages. Food Chem Toxicol 50: 2342-3428, 2012.

19. Granado-Serrano AB, Martín MÁ, Bravo L, et al: Quercetin attenuates TNF-induced inflammation in hepatic cells by inhibiting the NF- $\mathrm{BB}$ pathway. Nutr Cancer 64: 588-598, 2012.

20. Shen YC, Chen SL, Zhuang SR and Wang CK: Contribution of tomato phenolics to suppression of COX-2 expression in $\mathrm{KB}$ cells. J Food Sci 73: C1-C10, 2008.

21. Kim SH, Jun CD, Suk K, et al: Gallic acid inhibits histamine release and pro-inflammatory cytokine production in mast cells. Toxicol Sci 91: 123-131, 2006.

22. Chen $\mathrm{CH}$, Liu TZ, Chen $\mathrm{CH}$, et al: The efficacy of protective effects of tannic acid, gallic acid, ellagic acid, and propyl gallate against hydrogen peroxide-induced oxidative stress and DNA damage in IMR-90 cells. Mol Nutr Food Res 51: 962-968, 2007.

23. Arunkumar S, Ilango K, Manikandan RS and Ramalakshmi N: Synthesis and anti-inflammatory activity of some novel pyrazole derivatives of gallic acid. J Chem 6 (Suppl 1): S123-S128, 2009. 